\title{
Three Non-Pilot based Time- and Frequency Estimators for OFDM*
}

\author{
Jan-Jaap van de Beek ${ }^{1} \quad$ Per Ola Börjesson ${ }^{1} \quad$ Marie-Laure Boucheret $^{2}$ \\ Daniel Landström ${ }^{1}$ Julia Martinez Arenas ${ }^{2}$ \\ Per Ödling ${ }^{1}$ Sarah Kate Wilson ${ }^{1}$ \\ Elsevier's Signal Processing - Special Issue COST 254 workshop. \\ ${ }^{1}$ Luleå University of Technology, Division of Signal Processing, SE-971 87 Luleå, Sweden. \\ 2 Ecole National Supérieure de Télécommunications, Site de Toulouse, \\ BP4004 CEDEX, Toulouse, France.
}

\author{
Contact: \\ Jan-Jaap van de Beek \\ Division of Signal Processing \\ Luleå University of Technology \\ SE-971 87 Luleå, Sweden \\ tel: $\quad+4692072029$ \\ fax: $\quad+4692072043$ \\ e-mail: $\quad$ jaap@sm.luth.se
}

${ }^{*}$ This paper has been presented in part at the COST 254 Workshop, July 7-9, 1997, Toulouse, France, at the International Conference for Universal Personal Communications, October 13-16, 1997, San Diego, CA, USA and at Gretsi, September 15-19, 1997, Grenoble, France. 


\begin{abstract}
Time-domain Maximum-Likelihood (ML) estimators of time and frequency offsets are derived for three Orthogonal Frequency Division Multiplexing (OFDM) signal models: a pulse-shaped one-shot OFDM signal, a stream of multiple OFDM signals and an OFDM signal in a dispersive channel environment. We then develop structures to simplify their implementation. Simulation results show the relative performance and strengths of each of these three estimators.
\end{abstract}

\title{
1 Introduction
}

In this paper we focus on the estimation of time and frequency offsets for Orthogonal Frequency-Division Multiplexing (OFDM) symbols for synchronization purposes in wireless environments. The synchronization of an OFDM transmitter and receiver is important because OFDM systems, are in general more sensitive to time and frequency offsets than single carrier systems [17]. Not only may synchronization errors cause intersymbol-interference (ISI), they also can cause the loss of orthogonality between the subcarriers resulting in intercarrier-interference (ICI). The sensitivity of OFDM systems to synchronization errors has been documented in, e.g., [16][27][17][7]. Most time and frequency estimators for OFDM require pilot symbols, for example [3][26][14]. However, too many pilot symbols can lower the overall information rate. Hence methods that do not use pilots are desirable. Such methods have been investigated in [4][5][19][20][23][24] and patented in [19]. The method described in [23] differs from the previous methods in that it describes the time-domain maximum-likelihood (ML) estimator for OFDM systems with a cyclic-prefix [15] in an additive white Gaussian noise (AWGN) channel. This estimator not only exploits the redundancy in the cyclic-prefix, but also the relative power of the received signal to determine both the time and frequency offset of the received OFDM symbol. One of the main contributions of this paper is the knowledge that the estimator is optimal in the ML sense and as such gives an upper bound on the performance of a time and frequency estimator.

However, the estimator in [23] was derived for the case of a static AWGN channel and 
as such it is not necessarily suitable for wireless OFDM channels. This paper describes three signal models for wireless OFDM systems and derives the ML time and frequency estimator associated with each. We focus on the role of the signal model, rather than on a particular application. The goal of the paper is to provide a toolbox of techniques that can be used to improve the performance of the estimator from [23] by adapting the signal model to more accurately model the system. The purpose is also to build knowledge about the structure of ML estimators for OFDM systems so that one can use this knowledge to design for a specific system.

The three cases are as follows. First, pulse shaping is used in many wireless OFDM systems to reduce the out-of-band emission [8][11][21]. Because the ML estimator in [23] was derived for a non-pulse shaped OFDM system, it will suffer when applied to a pulse-shaped OFDM signal. We present an estimator for pulse-shaped systems [10][13]. Secondly, the ML estimator's performance suffers below a given SNR threshold [23]. For example, for bandlimited signals, proposed for multiuser communication systems [25], this threshold may reduce the applicability of the estimation concept. Here, we propose an estimator for a stream of OFDM symbols that can help compensate for such an SNR threshold. Finally, wireless OFDM systems often operate in a multipath environment, and thus under a dispersive channel [9]. Applying the estimator derived in [23][19] will result in a error floor in the time and frequency offset estimation. We develop an ML estimator that is based knowledge of the channel dispersion.

This paper is organized as follows, in Section 2 we discuss general properties of the OFDM system that are used in the estimation procedure. In Section 3 we present three signal models and the associated ML estimators. First we present the ML estimator for time and frequency offsets for systems using pulse shaping. Secondly, we present the ML estimator of time and frequency offsets for systems in which these parameters vary slowly. Finally, we present the ML estimator of a time offset for systems with channel dispersion. The paper does not target a particular application. Instead we will be working with signal models for a class of estimators and show how different system properties can be incorporated in the model. In Section 4 we illustrate the performance of the estimators with simulation results and we discuss these in Section 5. We show that 
by incorporating system properties as pulse shaping or knowledge about the stability of the clocks and oscillators, the estimator performance can significantly be improved.

\section{OFDM systems and synchronization}

Figure 1 shows the structure of the OFDM system on which our signal models are based. The transmitter modulates complex data symbols on a large number of carriers

Figure 1 by means of an inverse discrete Fourier transform (DFT). Each block of samples is cyclically extended with a prefix before it is transmitted over the channel [15]. In the receiver the cyclic prefix is removed and the data are demodulated by means of a DFT. If the cyclic prefix is longer than the length of the channel impulse response, it avoids ISI and ICI [15].

For the synchronization concept described in this paper the number of subcarriers $N$ and the length of the cyclic prefix $L$ are important parameters. They describe the amount of redundancy in the signal that the estimator can exploit. Reference [23] gives a thorough investigation of the estimator's performance in relation to $N$ and $L$.

The sensitivity to a symbol-time offset of $\theta$ samples has been investigated in [7]. As long as the time offset $\theta$ and the length of the channel impulse response together are smaller than half of the length of the cyclic prefix $L$, ISI and ICI are avoided. The time offset will then appear as phase offsets of the demodulated data symbols and a channel estimator can not distinguish these from channel phase distortions. The phase offsets will in a coherent system be compensated for in a channel equalizer and the system performance depends on the performance of the channel estimator and equalizer. In a differential system the system performance depends on how fast the channel is varying. For larger time offsets ISI and ICI occur [7]. Symbol timing requirements are relaxed by increasing the length of the cyclic prefix.

The sensitivity to frequency offsets for the AWGN channel has analytically been investigated in, among others, [17]. A frequency offset $\varepsilon$ (normalized to the intercarrier spacing) results in ICI. The amount of ICI is proportional to $\varepsilon^{2}$ and the signal-to-noise ratio (SNR). In [17] the ICI is interpreted as a degradation of the SNR and quantitatively 
investigated. For example, a frequency offset which is $4 \%$ of the intercarrier spacing results in interference that decreases the SNR with $0.2 \mathrm{~dB}$, if the original working SNR is $10 \mathrm{~dB}$, for a AWGN channel.

In the next section we discuss three models of the received OFDM signal. In each model we assume that the transmitted discrete-time signal is Gaussian [6]. Because of the cyclic prefix this signal is not white. Each transmitted OFDM symbol contains $L$ consecutive samples which are pairwise correlated with $L$ other consecutive samples, $N$ samples ahead. By observing this correlation one may tell where the OFDM symbol is likely to start. As we will see in the rest of the paper, the frequency offset can also be estimated exploiting this redundancy. Thus, we assume that the transmitted signal $s(k)$ is Gaussian with covariance function

$$
C_{s}\left(k_{1}, k_{2}\right)=\left\{\begin{aligned}
& \text { if } k_{1}=k_{2}, \\
& \sigma_{s}^{2}, \quad \text { if } k_{1}=k_{2}+N \text { and } 0 \leq k_{2}<L \\
& \text { if } k_{2}=k_{1}+N \text { and } 0 \leq k_{1}<L \\
& 0, \quad \text { otherwise. }
\end{aligned}\right.
$$

Notice that this model only reflects the appearance of one OFDM symbol (one cyclic prefix) in the transmitted signal. In a real system the transmitted signal consists of a stream of OFDM symbols, each containing this redundancy.

Based on the above assumptions, in [23] the received signal $r(k)$ is modelled as

$$
r(k)=s(k-\theta) e^{-j 2 \pi \varepsilon k / N}+n(k) \quad-\infty<k<\infty,
$$

where $s(k)$ is a sample of the Gaussian process with covariance function $(1), n(k)$ is complex AWGN with variance $\sigma_{n}^{2}$. We will compare the signal models developed in this paper with our reference model (2). We focus on the estimation of the unknown offset parameters $\theta$ and $\varepsilon$ from the received data $r(k)$. It is possible to estimate $\theta$ and $\varepsilon$ from $r(k)$, because much of the statistical structure of the transmitted signal $s(k)$ is transferred to the received signal $r(k)$.

In case the transmitter does not employ pulse-shaping and there is no channel dispersion, model (2) applies. The ML estimator based on (2) yields a fast one-shot estimator 
of $\theta$ and $\varepsilon$ [23]. We repeat the result from [23] here for later reference

$$
\begin{aligned}
& \widehat{\theta}_{0}=\arg \max _{\theta}\left\{\left|\gamma_{N}(\theta)\right|+\gamma_{0}(\theta)\right\}, \\
& \widehat{\varepsilon}_{0}=-\frac{1}{2 \pi} \measuredangle \gamma_{N}\left(\widehat{\theta}_{0}\right),
\end{aligned}
$$

where $\widehat{\theta}_{0}$ is the symbol-time offset, $\widehat{\varepsilon}_{0}$ is the frequency offset, and

$$
\begin{aligned}
\gamma_{N}(m) & =2 \sum_{k=m}^{m+L-1} r^{*}(k) r(k+N), \\
\gamma_{0}(m) & =-\rho \sum_{k=m}^{m+L-1}|r(k)|^{2}+|r(k+N)|^{2},
\end{aligned}
$$

and where

$$
\rho=\frac{\mathrm{SNR}}{\mathrm{SNR}+1}
$$

This estimator extracts the information carried by the cyclic prefix by correlating the received signal and a delayed version. The term $\gamma_{N}(m)$ collects this correlation and the term $\gamma_{0}(m)$ compensates for high contributions due to large samples rather than a large correlation.

\section{Estimators of time and frequency offsets}

In this section we generalize model (2) and estimator (3) to include pulse shaping, multiple symbols, and channel dispersion. We introduce the vector $\mathbf{r}$ for the received signal, with covariance matrix $\mathbf{C}_{\mathrm{r}}$. Then, the covariance function of the received signal contains the information due to (multiple) cyclic prefixes, pulse shaping or channel dispersion and noise. The joint maximum likelihood estimate $\widehat{(\theta, \varepsilon)}_{\mathrm{ML}}$ of $\theta$ and $\varepsilon$, given the received Gaussian data vector $\mathbf{r}$ with known covariance matrix $\mathbf{C}_{\mathrm{r}}$ becomes

$$
\widehat{(\theta, \varepsilon)}_{\mathrm{ML}}=\arg \max _{\theta, \varepsilon}\left\{-\mathbf{r}^{\mathrm{H}} \mathbf{C}_{\mathrm{r}}^{-1}(\theta, \varepsilon) \mathbf{r}\right\}
$$

For the signal models described in this section, the quadratic form can be put in an explicit expression which leads to implementable structures. 


\subsection{Offset estimators for pulse shaped systems}

Some OFDM systems require pulse shaping in order to suppress the system's sidelobes and out-of-band emission. The use of pulse shaping is suggested in, e.g., [7][8][11][21][12]. Examples of pulse shapes found in existing systems or system proposals are (see Figure 3) the Hanning pulse,

$$
p_{\mathrm{H}}(k)=\frac{1}{2}\left(1-\cos \left(\frac{2 \pi k}{N+L-1}\right)\right), \quad k=0 \leq k<N+L,
$$

investigated in [7][12], and the Tukey pulse,

$$
p_{\mathrm{T}}(k)= \begin{cases}\frac{1}{2}\left(1-\cos \left(\frac{k}{P-1} \pi\right)\right), & 0 \leq k<P \\ 1, & P \leq k<N+L-P, \\ \frac{1}{2}\left(1+\cos \left(\frac{k-(N+L-P)}{P-1} \pi\right)\right), & N+L-P \leq k<N+L,\end{cases}
$$

in [12]. For some pulse shapes and parameter choices, the tones in the OFDM symbol Figure 3 lose their orthogonality. The choice of pulse shapes is beyond the scope of this paper.

For a general pulse shape, we model the received signal as

$$
r(k)=g(k-\theta) s(k-\theta) e^{-j 2 \pi \varepsilon k / N}+n(k) \quad-\infty<k<\infty,
$$

where $\theta, \varepsilon, r(k), s(k)$, and $n(k)$ as in (2), and

$$
g(k)=\left\{\begin{array}{ll}
p(k) & 0 \leq k<N+L \\
1 & \text { otherwise }
\end{array},\right.
$$

where $p(k)$ is the pulse shape, for instance either (6) or (7). As in the reference model (2), we model knowledge (its correlation properties and its shape) for one OFDM symbol only ( $N+L$ consecutive samples) in the transmitted signal. Equation (8) models adjacent symbols as white Gaussian with time-invariant average power. For the choice $p_{\mathrm{R}}(k)=1, k=0 \leq k<N+L$, model (8) reduces to (2).

Pulse shaping affects the performance of an estimator in two ways, one negative and one positive. First, it will change the amplitude in some parts of the signal. For most practical pulses it reduces the amplitude in the parts of the signal that are cyclically repeated. This reduces the correlation in the signal, and thus also the performance of 
the estimator. Secondly, the reduction in amplitude introduces a time-varying signal power that also carries information about the symbol time offset. This information may improve the performance of an estimator. For some systems, the net effect of the pulse shaping is positive, as simulations will show.

To derive the ML estimator for the pulse-shaped OFDM system, we find the pair $\widehat{(\theta, \varepsilon)}$ that maximizes the $\log$-likelihood function $\Lambda(\theta, \varepsilon)=-\mathbf{r}^{\mathrm{H}} \mathbf{C}_{\mathrm{r}}^{-1}(\theta, \varepsilon) \mathbf{r}$. In Appendix $\mathrm{A}$ it is shown that this function becomes

$$
\Lambda(\theta, \varepsilon)=\left|\gamma_{N}(\theta)\right| \cos \left\{\measuredangle \gamma_{N}(\theta)+2 \pi \varepsilon\right\}+\gamma_{0}(\theta)
$$

where

$$
\begin{aligned}
\gamma_{N}(m) & =\sum_{k=-\infty}^{\infty} h_{N}(k-m) r(k) r^{*}(k+N), \\
\gamma_{0}(m) & =\sum_{k=-\infty}^{\infty} h_{0}(k-m)|r(k)|^{2}
\end{aligned}
$$

and where

$$
\begin{aligned}
& h_{N}(k)=\left\{\begin{array}{ll}
\frac{2 \operatorname{SNR} g(k) g(k+N)}{\operatorname{SNR}\left(g^{2}(k)+g^{2}(k+N)\right)+1} & 0 \leq k<L \\
0 & \text { otherwise }
\end{array},\right. \\
& h_{0}(k)= \begin{cases}\frac{\operatorname{SNR}^{2}(k+N)+1}{\operatorname{SNR}\left(g^{2}(k)+g^{2}(k+N)\right)+1} & 0 \leq k<L \\
\frac{\mathrm{SNR}^{2}(k-N)+1}{\operatorname{SNR}\left(g^{2}(k)+g^{2}(k-N)\right)+1} & N \leq k<N+L . \\
\frac{1}{\operatorname{SNR}^{2}(k)+1} & \text { otherwise }\end{cases}
\end{aligned}
$$

The SNR is the ratio of the average signal energy to the average noise energy (SNR = $\left.\sigma_{s}^{2} / \sigma_{n}^{2}\right)$. The ML estimator maximizes the log-likelihood function and becomes

$$
\begin{aligned}
& \widehat{\theta}_{1}=\arg \max _{\theta}\left\{\left|\gamma_{N}(\theta)\right|+\gamma_{0}(\theta)\right\}, \\
& \widehat{\varepsilon}_{1}=-\frac{1}{2 \pi} \measuredangle \gamma_{N}\left(\widehat{\theta}_{1}\right) .
\end{aligned}
$$

The filter $h_{0}(k)$ has infinite length. In order to make the filter length finite, we subtract the constant $\frac{1}{\sigma_{n}^{2}} \frac{1}{\operatorname{SNR}+1} \sum_{k=-\infty}^{\infty}|r(k)|^{2}$ from $\gamma_{0}(\theta)$. This is equivalent to subtracting it from the log-likelihood function $\Lambda(\theta, \varepsilon)$. Since this term does not depend on $\theta$ it will not change the maximizing argument $\widehat{\theta}_{1}$ (thus, there is no performance loss). Subtracting this constant from the log-likelihood function is equivalent to redefining $h_{0}(k)$ 
as

$$
h_{0}(k)=\left\{\begin{array}{ll}
\rho \frac{\mathrm{SNR}^{2}(k+N)+1-g^{2}(k)}{\operatorname{SNR}\left(g^{2}(k)+g^{2}(k+N)\right)+1} & 0 \leq k<L \\
\rho \frac{1-g^{2}(k)}{\operatorname{SNR}^{2}(k)+1} & L \leq k<N \\
\rho \frac{\operatorname{SNR}^{2}(k-N)+1-g^{2}(k)}{\operatorname{SNR}\left(g^{2}(k)+g^{2}(k-N)\right)+1} & N \leq k<N+L \\
0 & \text { otherwise }
\end{array},\right.
$$

where $\rho$ is as in estimator (3). This filter now has finite length and is thus implementable. When $p(k)=1$, the signal model (8) reduces to $(2)$ and the estimates $\widehat{\theta}_{1}$ and $\widehat{\varepsilon}_{1}$ coincide with $\widehat{\theta}_{0}$ and $\widehat{\varepsilon}_{0}$. A similar estimator performing one-shot estimation in a time-division multiuser systems is investigated in [10][13][22]. Figure 4 shows the estimator structure. Figure 4

Estimator (11) exploits two types of information. First the correlation between the samples in the cyclic prefix is used, and collected by the filter $h_{N}(k)$. Moreover, the time-varying signal power contains information about $\theta$. The estimator extracts this information by means of the filter $h_{0}(k)$. This filter $h_{0}(k)$ functions much like a matched filter to $|r(k)|^{2}$.

\subsection{Offset estimators for systems with $M$ consecutive OFDM symbols}

The signal models (2) and (8) incorporate a priori knowledge about one transmitted OFDM symbol in the transmitted signal and use only $N+L$ samples in the estimation procedure. Estimators based on these models are one-shot estimators in the sense that they generate estimates of the time and frequency offset for each symbol by exploiting the information carried by only that symbol. In this section we describe a signal model that incorporates multiple OFDM symbols to improve the estimator performance. It benefits from the statistical structure of $M$ consecutive OFDM symbols.

We model the received signal $r(k)$ as

$$
r(k)=s(k-\theta) e^{-j 2 \pi \varepsilon k / N}+n(k) \quad-\infty<k<\infty,
$$

where $\theta, \varepsilon$, and $n(k)$ as before. Here we assume that the transmitted signal $s(k)$ has 
covariance function

$$
C_{s}\left(k_{1}, k_{2}\right)=\left\{\begin{aligned}
& \text { if } k_{1}=k_{2} \\
\sigma_{s}^{2} \quad & \text { if } k_{1}=k_{2}+N \text { and } m(N+L) \leq k_{2}<m(N+L)+L \text { and } m=0 \ldots M \\
& \text { if } k_{2}=k_{1}+N \text { and } m(N+L) \leq k_{1}<m(N+L)+L \text { and } m=0 \ldots M \\
0 \quad & \text { otherwise }
\end{aligned}\right.
$$

The purpose of this model is to increase the information available to the estimator and to generate a stable symbol clock. In practical applications there may be a drift in the transmitter symbol clock relative to the receiver clock. In particular when $M$ is large, not all of the received symbols obey the perfect clock we assume. For instance, in systems with a multiple access scheme based on time-division, the drift from one symbol to the next may be large. In most applications, however, clock drifts are such that for moderate $M$, the assumption holds.

In appendix $\mathrm{B}$, we derive the $\log$-likelihood function of $\theta$ and $\varepsilon$ to get

$$
\Lambda(\theta, \varepsilon)=\left|\Gamma_{N}(\theta)\right| \cos \left\{\measuredangle \Gamma_{N}(\theta)+2 \pi \varepsilon\right\}+\Gamma_{0}(\theta)
$$

where

$$
\begin{aligned}
\Gamma_{N}(i) & =\sum_{m=0}^{M-1} \gamma_{N}(i+m(N+L)), \\
\Gamma_{0}(i) & =\sum_{m=0}^{M-1} \gamma_{0}(i+m(N+L)),
\end{aligned}
$$

and where $\gamma_{N}(m)$ and $\gamma_{0}(m)$ are as defined in (4). The optimal estimator for model (12) now becomes

$$
\begin{aligned}
& \widehat{\theta}_{2}=\arg \max _{\theta}\left\{\left|\Gamma_{N}(\theta)\right|+\Gamma_{0}(\theta)\right\}, \\
& \widehat{\varepsilon}_{2}=-\frac{1}{2 \pi} \measuredangle \Gamma_{N}\left(\widehat{\theta}_{2}\right) .
\end{aligned}
$$

Thus the optimal way (in a maximum likelihood sense) to process the $M$ consecutive symbols is to average the $M$ functions $\gamma_{N}(\cdot)$ and $\gamma_{0}(\cdot)$ in (4) to create the log-likelihood function. The estimator (14) can, in a straightforward way, be extended to incorporate pulse shaping as well. 


\subsection{Offset estimators for systems with channel dispersion}

In many applications, for instance the European Digital Audio Broadcasting (DAB) network [1], the Digital Video Broadcasting (DVB) [2] and the Universal Mobile Telecommunications System (UMTS) [25] dispersion in the channel will affect the correlation properties of the received OFDM signal. For the purpose of data detection in an OFDM receiver, this channel dispersion appears as a multiplicative distortion of the data symbols and correction is straightforward. However, the time and frequency synchronization concept targeted by this paper works on the received signal $r(k)$ before the DFT. In this section we state a signal model incorporating $r(k)$ 's correlation due to the channel dispersion. As we will see though, optimal synchronization which is conceptually straightforward, is not as tractable as in the previous cases and some approximations are necessary to reach an implementable estimator.

Given a channel impulse response, $h(k)$, we model the received signal $r(k)$ as

$$
r(k)=(h * s)(k-\theta) e^{-j 2 \pi \varepsilon k / N}+n(k) \quad-\infty<k<\infty,
$$

where $\theta, \varepsilon, s(k)$ and $n(k)$ are as defined above. We assume that the length $H$ of $h(k)$ is smaller than the length of the cyclic prefix $L$. Because of the dispersive channel, $h(k)$, the correlation structure of $s(k)$ is not transferred so directly to the received signal $r(k)$. Although the channel colors the received signal, we will show that estimation is possible.

We assume that we know the channel impulse response. While this assumption holds for some applications (such as copper wire channels), the receiver will have to track a time-varying channel in others (such as DAB, DVB, UMTS). However, this assumption and the following derivation will give an upper bound on the performance of an estimator that does not use pilots and is therefore useful when evaluating other estimator's performance.

As with the previous models, the ML estimator depends on the covariance matrix $\mathbf{C}_{\mathbf{r}}$ of the received data vector $\mathbf{r}$. We can write this matrix as

$$
\mathbf{C}_{\mathbf{r}}=\mathbf{H C}_{\mathbf{s}} \mathbf{H}^{H}+\sigma_{n}^{2} \mathbf{I}
$$

where the matrix $\mathbf{C}_{\mathbf{s}}$ is the correlation matrix of the transmitted OFDM signal $s(k)$ 
and whose $\left(k_{1}, k_{2}\right)^{t h}$ entries are $C_{s}\left(k_{1}, k_{2}\right)(1)$, and $\mathbf{H}$ is a matrix whose entries are of the form, $\mathbf{H}_{i,(i: i+H-1)}=(h(0), h(1), \ldots, h(H-1))$. We can rewrite $\mathbf{C}_{\mathbf{r}}$ as the sum of three separate matrices,

$$
\mathbf{C}_{\mathbf{r}}=\mathbf{C}_{0}+\sigma_{n}^{2} \mathbf{I}+\mathbf{C}_{\mathrm{cp}}(\boldsymbol{\theta})
$$

The first term $\mathbf{C}_{0}$ is a band matrix representing the channel correlation. Specifically,

$$
\mathbf{C}_{0}=\sigma_{s}^{2} \mathbf{H H}^{\mathbf{H}} \text {. }
$$

The second term $\sigma_{n}^{2} \mathbf{I}$ is a matrix representing the noise correlation. The third term $\mathbf{C}_{\mathrm{cp}}(\boldsymbol{\theta})$ is a matrix representing the total correlation of samples located in the repeated parts, i.e., the cyclic prefix. That is, $\mathbf{C}_{\mathrm{cp}}(\boldsymbol{\theta})=\mathbf{H I}(\theta) \mathbf{H}^{\mathbf{H}}$, where

$$
\mathbf{I}(\theta)_{i, j}=\left\{\begin{array}{ll}
\sigma_{s}^{2} & \text { if }|j-i|=N+\theta, i \in[\theta, \theta+L-1] \\
\sigma_{s}^{2} & \text { if }|j-i|=N+\theta, j \in[\theta, \theta+L-1] \\
0 & \text { otherwise }
\end{array} .\right.
$$

Note that only $\mathbf{C}_{\mathrm{cp}}(\theta)$ depends on the unknown $\theta$. The only non-zero elements of $\mathbf{C}_{\mathrm{cp}}(\theta)$ are concentrated in a $(N+L+H) \times(N+L+H)$ submatrix, and the dependency of $\theta$ appears only in the position of this submatrix. The first and second terms form a bandmatrix $\mathbf{C}_{0}+\sigma_{n}^{2} \mathbf{I}$ that is independent of $\theta$.

The optimal estimator for model (15) is then

$$
\widehat{\theta}_{3}=\arg \max _{\theta}\left\{-\mathbf{r}^{H} \mathbf{C}_{\mathbf{r}}^{-1}(\theta) \mathbf{r}\right\}
$$

As the size of $\mathbf{C}_{\mathbf{r}}$ increases, so does the complexity of the ML operator. For this reason, we show a derivation of the ML estimator with a more manageable complexity. In our derivation, we present only $\widehat{\theta}_{2}$, ignoring the estimation of $\varepsilon$. Our motivation for this is twofold. First we want a tractable estimator that gives an indication of likely performance in a dispersive case so that we can gain an understanding of what synchronization information is contained in $r(k)$. Secondly, from previous applied work [23] we have seen that the performance of frequency estimators based on models (2) and (8) is sufficient in a dispersive channel, whereas the performance of the time estimators is not. Thus we focus on estimation of $\widehat{\theta}_{2}$. 
To simplify our estimator, we now define the matrix

$$
\mathbf{A}(\theta)=\mathbf{C}_{r}^{-1}(\theta)-\left(\mathbf{C}_{0}+\sigma_{n}^{2} \mathbf{I}\right)^{-1}
$$

Then, combining (16) and (17) we get

$$
\begin{aligned}
\widehat{\theta}_{3} & =\arg \max _{\theta}\left\{-\mathbf{r}^{H} \mathbf{A}(\theta) \mathbf{r}+\mathbf{r}^{H}\left(\mathbf{C}_{0}+\sigma_{n}^{2} \mathbf{I}\right)^{-1} \mathbf{r}\right\} \\
& =\arg \max _{\theta}\left\{-\mathbf{r}^{H} \mathbf{A}(\theta) \mathbf{r}\right\}
\end{aligned}
$$

since the maximizing argument does not depend on $\mathbf{r}^{H}\left(\mathbf{C}_{0}+\sigma_{n}^{2} \mathbf{I}\right)^{-1} \mathbf{r}$. Variations in $\theta$ shift the elements of $\mathbf{A}(\theta)$ along the diagonals. Substraction of $\mathbf{r}^{H}\left(\mathbf{C}_{0}+\sigma_{n}^{2} \mathbf{I}\right)^{-1} \mathbf{r}$ is the equivalent of the substraction of $\frac{1}{\sigma_{n}^{2}} \frac{1}{\operatorname{SNR}+1} \sum_{k=-\infty}^{\infty}|r(k)|^{2}$ in the derivation of the estimator for pulse shaping (11).

Using a matrix inversion lemma [18], we can show that

$$
\mathbf{A}(\theta)=-\left(\mathbf{C}_{0}+\sigma_{n}^{2} \mathbf{I}\right)^{-1} \mathbf{C}_{\mathrm{cp}}(\theta)\left(\mathbf{I}+\left(\mathbf{C}_{0}+\sigma_{n}^{2} \mathbf{I}\right)^{-1} \mathbf{C}_{\mathrm{cp}}(\theta)\right)^{-1}\left(\mathbf{C}_{0}+\sigma_{n}^{2} \mathbf{I}\right)^{-1}
$$

Because $\mathbf{C}_{\mathrm{cp}}(\theta)$ is a block matrix with zeros outside an $(N+H+L) \times(N+H+L)$ submatrix, the matrix $\mathbf{A}(\theta)$, which is direct product of other matrices and $\mathbf{C}_{\mathrm{cp}}(\theta)$, is approximately also limited to a $(N+H+L) \times(N+H+L)$ submatrix. Studying the behavior of the matrix $\mathbf{A}(\theta)$ for different communication scenarios gives some indication about how information about $\theta$ is contained in the received signal. Based on the observation that information about $\theta$ is largely concentrated in a relatively small section of $\mathbf{A}(\theta)$, we will proceed with the approximation of $\mathbf{A}(\theta)$ by a matrix $\widetilde{\mathbf{A}}(\theta)$ whose entries in positions outside the submatrix of size $(N+H+L) \times(N+H+L)$ are zero. Although the difference between the matrices may often be quite small, this does not necessarily mean that the estimator performance degradation is negligible. Rather, this is a means of getting a more practical estimator.

We can further simplify our time-offset estimator by noting that:

$$
\begin{aligned}
\Lambda(\theta) & \approx-\mathbf{r}^{H} \widetilde{\mathbf{A}}(\theta) \mathbf{r} \\
& =\sum_{k} \sum_{j} \mathbf{r}_{k}^{*} \widetilde{\mathbf{A}}_{k, j}(\theta) \mathbf{r}_{j} \\
& =\sum_{i} \sum_{k} \mathbf{r}_{k}^{*} \widetilde{\mathbf{A}}_{k-\theta, k-\theta+i}(0) \mathbf{r}_{k+i}
\end{aligned}
$$




$$
=\sum_{i} \sum_{l} \mathbf{r}_{l+\theta}^{*} \widetilde{\mathbf{A}}_{l, l+i}(0) \mathbf{r}_{l+\theta+i}
$$

where $\widetilde{\mathbf{A}}(0)$ is the $\theta$-independent matrix $\left.\widetilde{\mathbf{A}}(\theta)\right|_{\theta=0}$. Since all non-zero elements of $\widetilde{\mathbf{A}}(0)$ are concentrated in an $(N+L+H) \times(N+L+H)$ submatrix there are only $2(N+L+H)-1$ diagonals of $\widetilde{\mathbf{A}}(0)$ that contain non-zero elements. This result finally yields the time offset estimate

$$
\widehat{\theta}_{3}=\arg \max _{\theta}\left\{\sum_{i=-(N+L)}^{(N+L)} \gamma_{i}(\theta)\right\},
$$

where

$$
\gamma_{i}(m)=\sum_{k=-\infty}^{\infty} h_{i}(k-m) r(k) r^{*}(k+i),
$$

and where $h_{i}(k)$ is the negative of the $i$ th diagonal of $\widetilde{\mathbf{A}}(0)$. This result is important because one can now implement the estimator with filters $h_{i}(k)$. Filter $h_{i}(k)$ has finite support of length $(N+L+H)-|i|$. Estimator (18) suggests the implementation structure shown in Figure 5. First the products $r(k) r^{*}(k+i)$ are formed. These are Figure 5 fed into the filterbank containing filters with impulse responses $h_{i}(k)$ (the diagonals of $\widetilde{\mathbf{A}}(0))$.

Low-complexity approximations of (18) can be obtained by disregarding filters with a relatively small contribution to the quadratic form and just regarding the most significant diagonals. Typically, for a channel with a short impulse response, filters $h_{i}(k)$ with $i$ equal or close to $-N, 0$ and $N$ contribute most. In general, the complexity and the approximation error relative to the optimal estimator can be varied by varying the number of filters.

This estimator structure is similar to the structure (3). Without channel dispersion, only the terms $\gamma_{0}(m), \gamma_{-N}(m)$ and $\gamma_{N}(m)$ contain non-zero values and estimator (18) reduces to $(3)$ for $\varepsilon=0$. The estimate $\widehat{\theta}_{3}$ then coincides with $\widehat{\theta}_{0}$. Also, the estimator (18) can, in a straightforward way be extended to incorporate pulse shaping as well. The pulse shape appears in the covariance matrix $\mathbf{C}_{r}$ and in the diagonals of $\widetilde{\mathbf{A}}(0)$. If pulse shaping is incorporated, but used in an environment without channel dispersion (18) reduces to $(11)$. 


\section{Simulations}

\subsection{Performance in a system with pulse shaping}

We illustrate the estimator concepts described in the previous sections with the following system. Consider a fictitious OFDM system with 64 subcarriers. In order to reduce ISI and ICI in a dispersive channel environment the system employs a cyclic prefix of 16 samples. Also, we assume that the transmitter shapes 8 samples of an OFDM symbol (4 samples at each side) with the Tukey pulse shape.

We first investigate how the pulse shape in this system affects the estimator performance. Figure 6 shows the variance of the time-offset estimators (3), designed for no pulse shaping, and (11), designed for pulse shaping. Thus, one estimator does not take the pulse shape into account and one does. Both estimators are then extended with the averaging concept (14), yielding another two estimators (see Figure 6). Ignoring the pulse shape in the design of the estimator results in a performance error floor. This error floor can be decreased by averaging but consideration of the pulse shape in the estimator design removes this error floor. Figure 7 shows the estimator variance for the frequency estimators designed as described above. In our system scenario the inclusion of the pulse shape in the model does not significantly improve the performance of the frequency estimator.

\subsection{Performance in a dispersive environment}

We now assume that the system operates in a dispersive environment. The channel dispersion is a maximum of 8 samples. We model the channel with the following static discrete-time exponentially decaying channel impulse response

$$
h(\tau)=e^{-\tau / 3}, \quad \tau=0, \ldots, 7 .
$$

As above, the transmitter employs the Tukey pulse shape. Figure 8 shows the performance of the time offset estimators (3), designed with no knowledge of the pulse shape nor the channel dispersion, (11), designed with no knowledge of the channel dispersion 
but incorporating the pulse shape, and (18) for $\varepsilon=0$, designed without knowledge of the pulse shape but incorporating the channel dispersion. Thus, one estimator takes neither pulse shaping nor dispersion into account (reference estimator), one estimator takes the pulse shape only into account, and one estimator recognizes the channel dispersion only. In this scenario the performance of each estimator experiences an error floor because none of the estimators is perfectly matched to the true signal properties. Using knowledge of the pulse shape property improves the performance more than using knowledge of the channel dispersion alone.

Figures 9 and 10 show the performance of the joint estimators (3) (the reference estimator) and (11) (the pulse shaping estimator). Both estimators are also extended with the averaging concept (14). In our dispersive environment the use of estimator (11) combined with averaging decreases the error floor.

The simulation results in this section suggest that in systems with pulse shaping, estimator performance is increased by incorporating the pulse shape in the signal model. Moreover, in systems with channel dispersion, where time and frequency offsets vary slowly, averaging decreases the error floor.

\section{Summary}

We have presented three signal models and their respective ML estimators of time and frequency offsets for OFDM systems. Each of the models confronts a difficulty in estimating the time and frequency in a wireless OFDM system. When applied to a wireless system, all of the estimators can improve on the performance of the $\mathrm{ML}$ estimator designed for an AWGN channel [23]. We presented the ML estimator based on a signal model that considers the pulse shape. Simulations show that the use of this estimator in systems with pulse shaping is beneficial in both AWGN and dispersive environments. We have also found that when the clock drift is small, averaging can significantly improve the error floor of the estimator. The estimator designed for a dispersive channel can be quite complex, but is useful since it gives a bound on the achievable performance. 


\section{A The ML estimator for pulse shaping}

For model (8) the log-likelihood function $\Lambda(\theta, \varepsilon)$ can be written as

$$
\Lambda(\theta, \varepsilon)=\log \left(\prod_{k \in[\theta, \theta+L-1]} f(r(k), r(k+N)) \cdot \prod_{k \notin[\theta, \theta+L-1] \cup[\theta+N, \theta+N+L-1]} f(r(k))\right),
$$

where $f(r(k), r(k+N))$ is the joint Gaussian probability density function for the pair $(r(k), r(k+N)), k \in[\theta, \theta+L-1]$ (samples coupled through the cyclic extension), and $f(r(k))$ denotes the Gaussian density function for other values of $k$. Now, define $\mathbf{r}=\left[\begin{array}{ll}r(k) & r(k+N)\end{array}\right]^{T}$. By calculating the densities $f(r(k))$ and $f(\mathbf{r})$ we will find the $\log$-likelihood function $\Lambda(\theta, \varepsilon)$. First, $r(k)$ is a complex Gaussian variable with density $f(r(k))$ and

$$
\log f(r(k))=-\frac{|r(k)|^{2}}{\sigma_{s}^{2} g(k-\theta)^{2}+\sigma_{n}^{2}}+\log c_{1} \quad-\infty<k<\infty
$$

where $\sigma_{s}^{2} \equiv E\left\{|s(k)|^{2}\right\}$ and $\sigma_{n}^{2} \equiv E\left\{|n(k)|^{2}\right\}$. Secondly, $\mathbf{r}$ is a complex Gaussian vector with joint density function $f(\mathbf{r})$

$$
\log f(\mathbf{r})=-\mathbf{r}^{H} \mathbf{C}^{-1} \mathbf{r}+\log c_{2}
$$

and $\mathbf{C}$ its covariance matrix. $\mathbf{C}$ becomes

$\mathbf{C}=E\left\{\mathbf{r}^{H} \mathbf{r}\right\}=\sigma_{n}^{2}\left[\begin{array}{ll}\operatorname{SNR}^{2}(k-\theta)+1 & \operatorname{SNR} g(k-\theta) g(k+N-\theta) e^{-j 2 \pi \varepsilon} \\ \operatorname{SNR} g(k+N-\theta) g(k-\theta) e^{j 2 \pi \varepsilon} & \operatorname{SNR}^{2}(k+N-\theta)+1\end{array}\right]$.

Thus,

$$
\begin{aligned}
\log f(\mathbf{x})= & -\frac{1}{D}\left(\operatorname{SNR}^{2}(k+N-\theta)+1\right)|r(k)|^{2}-\frac{1}{D}\left(\operatorname{SNR}^{2}(k-\theta)+1\right)|r(k+N)|^{2} \\
& +\frac{2}{D} \operatorname{SNR} g(k-\theta) g(k+N-\theta) \operatorname{Re}\left\{e^{-j 2 \pi \varepsilon} r^{*}(k) r(k+N)\right\}
\end{aligned}
$$

where $D$ is the discriminant of $\mathbf{C}$,

$$
D=\sigma_{n}^{2}\left(\operatorname{SNR}\left(g^{2}(k-\theta)+g^{2}(k+N-\theta)\right)+1\right) .
$$

Straightforward calculations now yield 


$$
\begin{aligned}
\Lambda(\theta, \varepsilon) & =\sum_{k \in[\theta, \theta+L-1]} \log f(r(k), r(k+N))+\sum_{k \notin[\theta, \theta+L-1] \cup[\theta+N, \theta+N+L-1]} \log f(r(k)) \\
& =\sum_{k=\theta}^{\theta+L-1} h_{N}(k-\theta) \operatorname{Re}\left\{e^{-j 2 \pi \varepsilon} r^{*}(k) r(k+N)\right\}+\sum_{k=\theta}^{\theta+L-1} h_{0}(k-\theta)|r(k)|^{2},
\end{aligned}
$$

where $h_{N}(k)$ and $h_{0}(k)$ defined in (10). Finally

$$
\Lambda(\theta, \varepsilon)=\left|\gamma_{N}(\theta)\right| \cos \left\{\measuredangle \gamma_{N}(\theta)+2 \pi \varepsilon\right\}+\gamma_{0}(\theta),
$$

where $\gamma_{N}(\theta)$ and $\gamma_{0}(\theta)$ as defined in $(9)$.

\section{B The ML estimator for $M$ consecutive symbols}

Assume that the observation interval contains $M$ complete OFDM symbols. The arrival time $\theta$ is, as before, the index of the first sample of the first complete symbol, modelling the unknown channel delay. Consider the cyclic prefixes $\mathcal{I}_{m}$ and their copies $\mathcal{I}_{m}^{\prime}$ for each symbol $m=0, \ldots, M-1$ :

$$
\begin{aligned}
& \mathcal{I}_{m} \equiv\{m(N+L)+\theta, \ldots, m(N+L)+\theta+L-1\}, \\
& \mathcal{I}_{m}^{\prime} \equiv\{m(N+L)+\theta+N, \ldots, m(N+L)+\theta+N+L-1\},
\end{aligned}
$$

and define the union of all these indexes

$$
\begin{aligned}
\mathcal{I} & \equiv \bigcup_{m=0}^{M-1} \mathcal{I}_{m}, \\
\mathcal{I}^{\prime} & \equiv \bigcup_{m=0}^{M-1} \mathcal{I}_{m}^{\prime} .
\end{aligned}
$$

The observation samples $r(k)$ can now be divided into the samples $r(k), k \in \mathcal{I} \cup \mathcal{I}^{\prime}$, which are pairwise dependent, and the remaining samples $r(k), k \notin \mathcal{I} \cup \mathcal{I}^{\prime}$ which are independent. Using these properties, the probability density function of the observation can be written as

$$
\begin{aligned}
f(\mathbf{r} \mid \theta, \varepsilon)= & \prod_{k \in \mathcal{I}} f(r(k), r(k+N)) \prod_{k \notin \mathcal{I}} f(r(k))= \\
& \prod_{k \in \mathcal{I}} \frac{f(r(k), r(k+N))}{f(r(k)) f(r(k+N))} \prod_{k} f(r(k)) .
\end{aligned}
$$


The last factor is independent of $\theta$ and $\varepsilon$ and can thus be omitted. The remaining part can be rewritten because of the independence of samples from different symbols

$$
\prod_{k \in \mathcal{I}} \frac{f(r(k), r(k+N))}{f(r(k)) f(r(k+N))}=\prod_{m=0}^{M-1}\left(\prod_{k \in \mathcal{I}_{m}} \frac{f(r(k), r(k+N))}{f(r(k)) f(r(k+N))}\right) .
$$

Using the statistical properties of $r(k)$ and following the lines of appendix A, the $\log$ likelihood function given the observation of $M$ symbols now becomes

$$
\begin{aligned}
\Lambda(\theta, \varepsilon)= & \sum_{m=0}^{M-1} \sum_{k=\theta}^{\theta+L-1} \operatorname{Re}\left\{e^{-j 2 \pi \varepsilon} r^{*}(k+m(N+L)) r(k+m(N+L)+N)\right\} \\
& +\sum_{m=0}^{M-1} \sum_{k=\theta}^{\theta+L-1}|r(k+m(N+L))|^{2} \\
= & \left.\left|\Gamma_{N}(\theta)\right| \cos \left\{\measuredangle \Gamma_{N}(\theta)+2 \pi \varepsilon\right\}+\Gamma_{0}(\theta)\right),
\end{aligned}
$$

where $\Gamma_{N}(\theta)$ and $\Gamma_{0}(\theta)$ are defined in (13).

\section{References}

[1] Radio broadcasting systems; Digital Audio Broadcasting (DAB) to mobile, portable and fixed receivers. ETS 300 401, ETSI - European Telecommunications Standards Institute, Valbonne, France, Feb. 1995.

[2] Digital broadcasting systems for television, sound and data services. European Telecommunications Standard, prETS 300 744, Sept. 1996.

[3] F. Classen and H. Meyr. Frequency synchronization algorithms for OFDM systems suitable for communication over frequency-selective fading channels. In Proc. IEEE Vehic. Technol. Conf., volume 3, pages 1655-1659, Stockholm, Sweden, June 1994.

[4] F. Daffara and O. Adami. A new frequency detector for orthogonal multicarrier transmission techniques. In Proc. IEEE Vehic. Technol. Conf., volume 2, pages 804-809, Chicago, IL, July 1995.

[5] F. Daffara and A. Chouly. Maximum-likelihood frequency detectors for orthogonal multicarrier systems. In Proc. Intern. Conf. Commun., pages 766-771, Geneva, Switzerland, May 1993. 
[6] R. Gross and D. Veeneman. Clipping distortion in DMT ADSL systems. Electron. Lett., 29(24):2080-2081, Nov. 1993.

[7] M. Gudmundson and P.-O. Andersson. Adjacent channel interference in an OFDM system. In Proc. IEEE Vehic. Technol. Conf., volume 2, pages 918-922, Atlanta, GA, Apr. 1996.

[8] R. Haas. Application des transmissions à porteuses multiples aux communications radio mobiles. Phd. thesis, Ecole National Supérieure des Télécommunications, Paris, France, Jan. 1996. In English.

[9] W. C. Jakes. Microwave mobile communications. Classic Reissue. IEEE Press, Piscataway, New Jersey, 1974.

[10] D. Landström, J. Martinez Arenas, J.-J. van de Beek, P. O. Börjesson, M.-L. Boucheret, and P. Ödling. Time and frequency offset estimation in OFDM systems employing pulse shaping. In Proc. Int. Conf. Universal Personal Commun., pages 279-283, San Diego, CA, USA, Oct. 1997.

[11] B. Le Floch, M. Alard, and C. Berrou. Coded orthogonal frequency-division multiplexing. Proc. IEEE, 83(6):982-996, June 1995.

[12] G. Malmgren. Single Frequency Broadcasting Networks. PhD thesis, Royal Institute of Technology, Stockholm, Sweden, Apr. 1997.

[13] J. Martinez Arenas, D. Landström, J.-J. van de Beek, P. O. Börjesson, M.-L. Boucheret, and P. Ödling. Performance of a joint time and frequency offset estimator for different pulse shapes in OFDM systems. In GRETSI, Grenoble, France, Sept. 1997.

[14] P. Moose. A technique for orthogonal frequency-division multiplexing frequency offset correction. IEEE Trans. Commun., 42(10):2908-2914, Oct. 1994. 
[15] A. Peled and A. Ruiz. Frequency domain data transmission using reduced computational complexity algorithms. In Proc. IEEE Int. Conf. Acoust., Speech, Signal Processing, pages 964-967, Denver, CO, 1980.

[16] T. Pollet and M. Moeneclaey. Synchronizability of OFDM signals. In Proc. Globecom, volume 3, pages 2054-2058, Singapore, Nov. 1995.

[17] T. Pollet, M. van Bladel, and M. Moeneclaey. BER sensitivity of OFDM systems to carrier frequency offset and Wiener phase noise. IEEE Trans. Commun., 43(2/3/4):191-193, Feb/Mar/Apr 1995.

[18] L. L. Scharf. Statistical signal processing: Detection, estimation, and time series analysis. Addison-Wesley, 1991.

[19] T. Seki, Y. Sugita, and T. Ishikawa. Ofdm synchronization demodulation unit. United States Patent, (5,602,835), Feb. 1997.

[20] P. J. Tourtier, R. Monnier, and P. Lopez. Multicarrier modem for digital HDTV terrestrial broadcasting. Signal Proc.: Image Commun., 5(5-6):379-403, Dec. 1993.

[21] A. Vahlin and N. Holte. Optimal finite duration pulses for OFDM. IEEE Trans. Commun., 44(1):10-14, Jan. 1996.

[22] J.-J. van de Beek, P. O. Börjesson, M. L. Boucheret, D. Landström, J. Martinez Arenas, and P. Ödling. An all-digital symbol synchronizer in an OFDM modem for wireless comunication. In Proc. COST 254, Toulouse, France, July 1997. Submitted to.

[23] J.-J. van de Beek, M. Sandell, and P.-O. Börjesson. ML estimation of timing and frequency offset in OFDM systems. IEEE Trans. Signal Proc., 45(7):1800-1805, July 1997.

[24] J.-J. van de Beek, M. Sandell, M. Isaksson, and P. O. Börjesson. Low-complex frame synchronization in OFDM systems. In Proc. Int. Conf. Universal Personal Commun., pages 982-986, Tokyo, Japan, Nov. 1995. 
[25] M. Wahlqvist et al. Description of Telias OFDM based proposal (working document in the OFDM concept group). Technical Report Tdoc 180/97, ETSI STC SMG2 meeting no 22, Bad Aibling, Germany, May 1997.

[26] W. D. Warner and C. Leung. OFDM/FM frame synchronization for mobile radio data communication. IEEE Trans. Vehic. Technol., 42(3):302-313, Aug. 1993.

[27] L. Wei and C. Schlegel. Synchronization requirements for multi-user OFDM on satellite mobile and two-path Rayleigh-fading channels. IEEE Trans. Commun., 43(2/3/4):887-895, Feb/Mar/Apr 1995. 


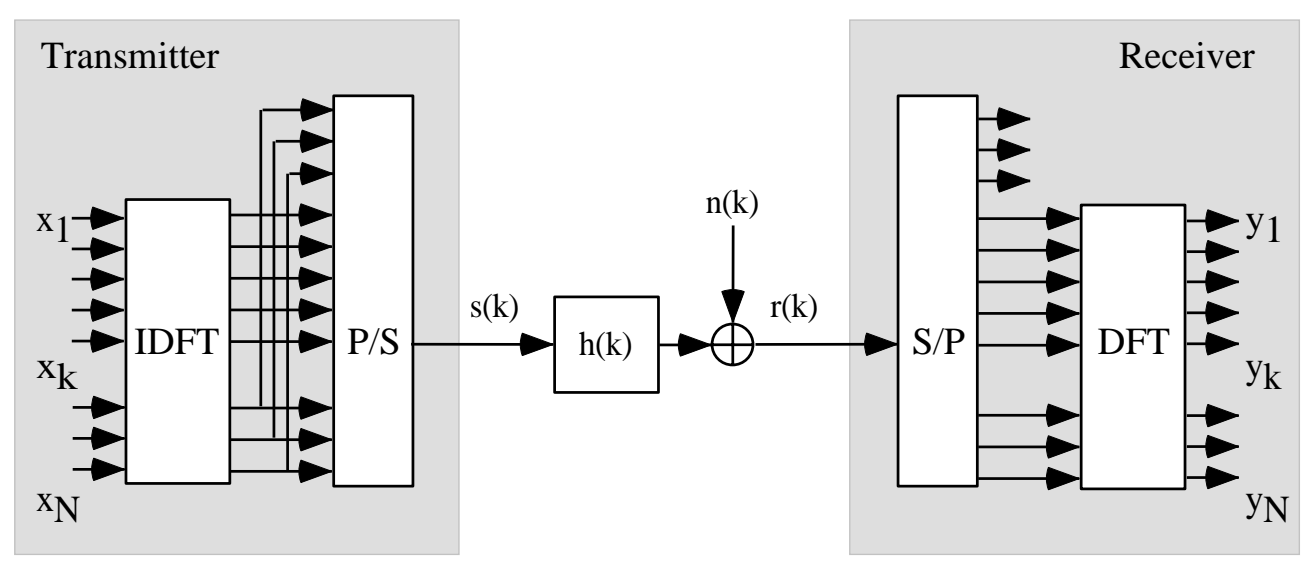

Figure 1: The OFDM system model. 


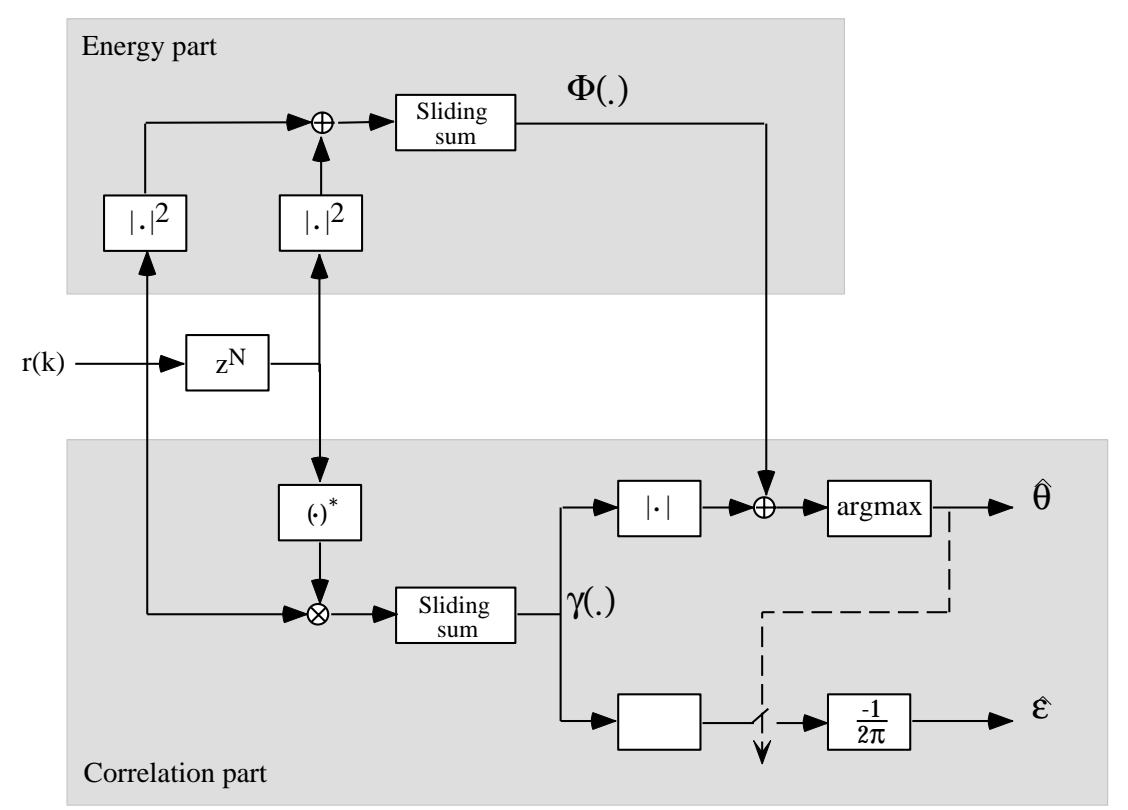

Figure 2: Structure of the estimator designed for an AWGN channel.

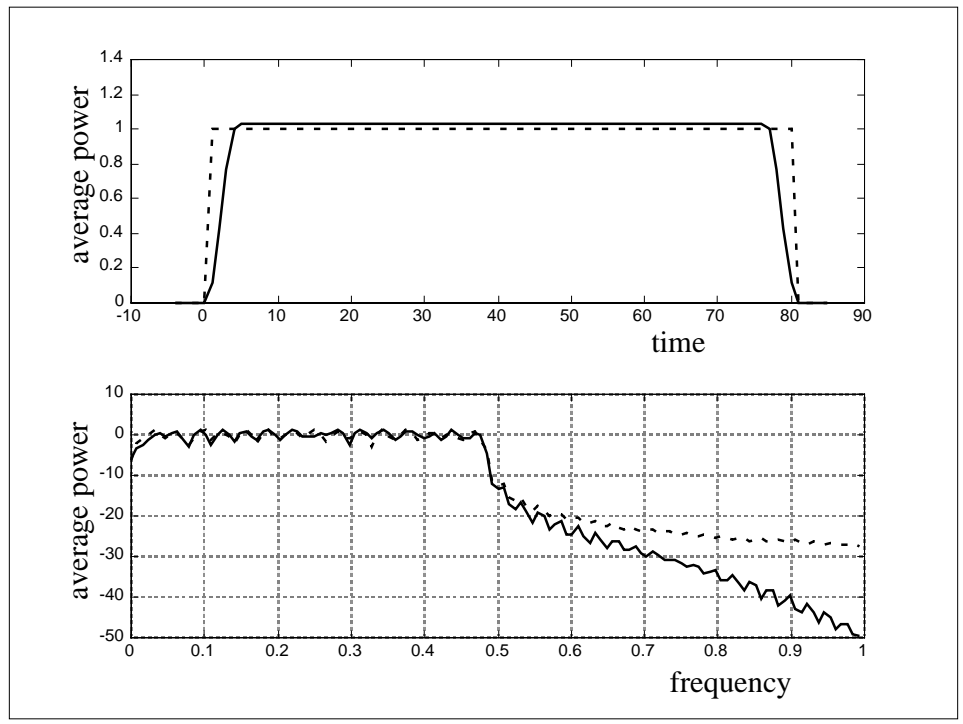

Figure 3: Spectrum of pulse shaped OFDM signals. Rectangular pulse (dashed) and Tukey pulse (solid). 


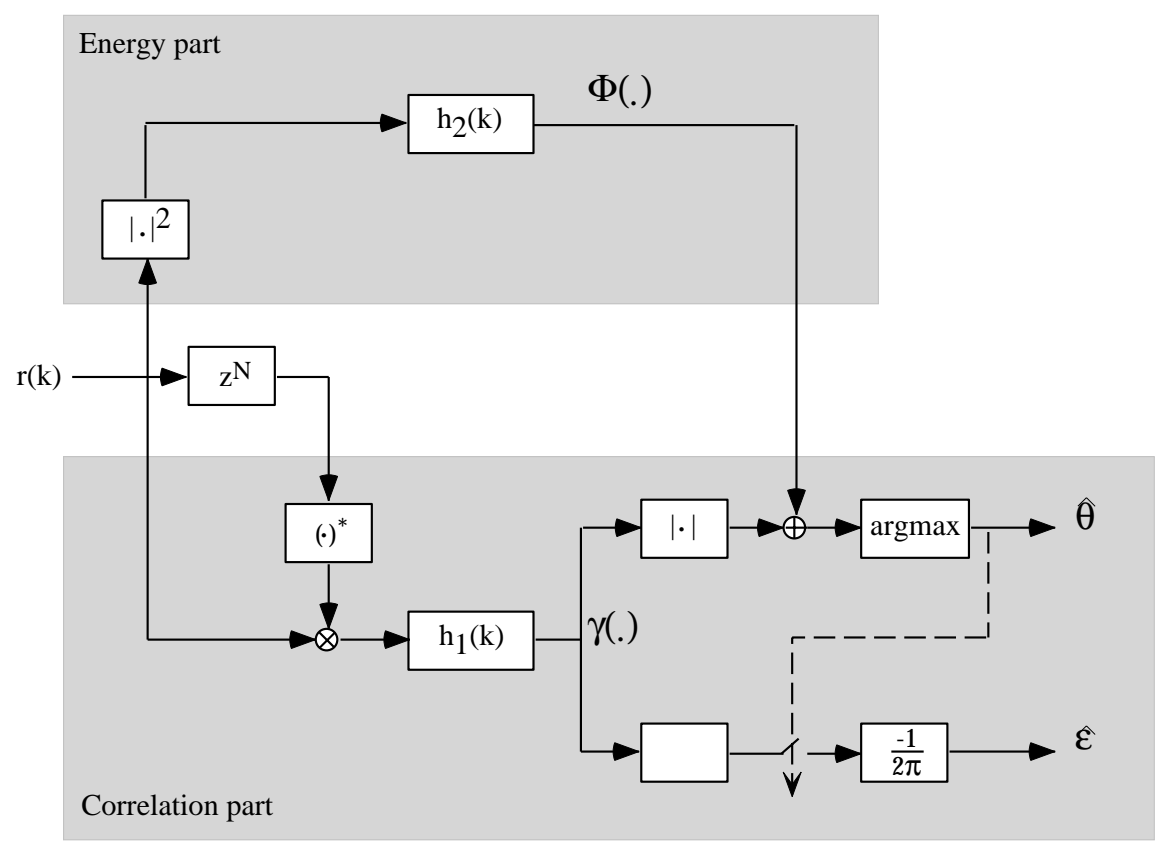

Figure 4: Structure of the estimator designed for the AWGN channel and pulse shaping.

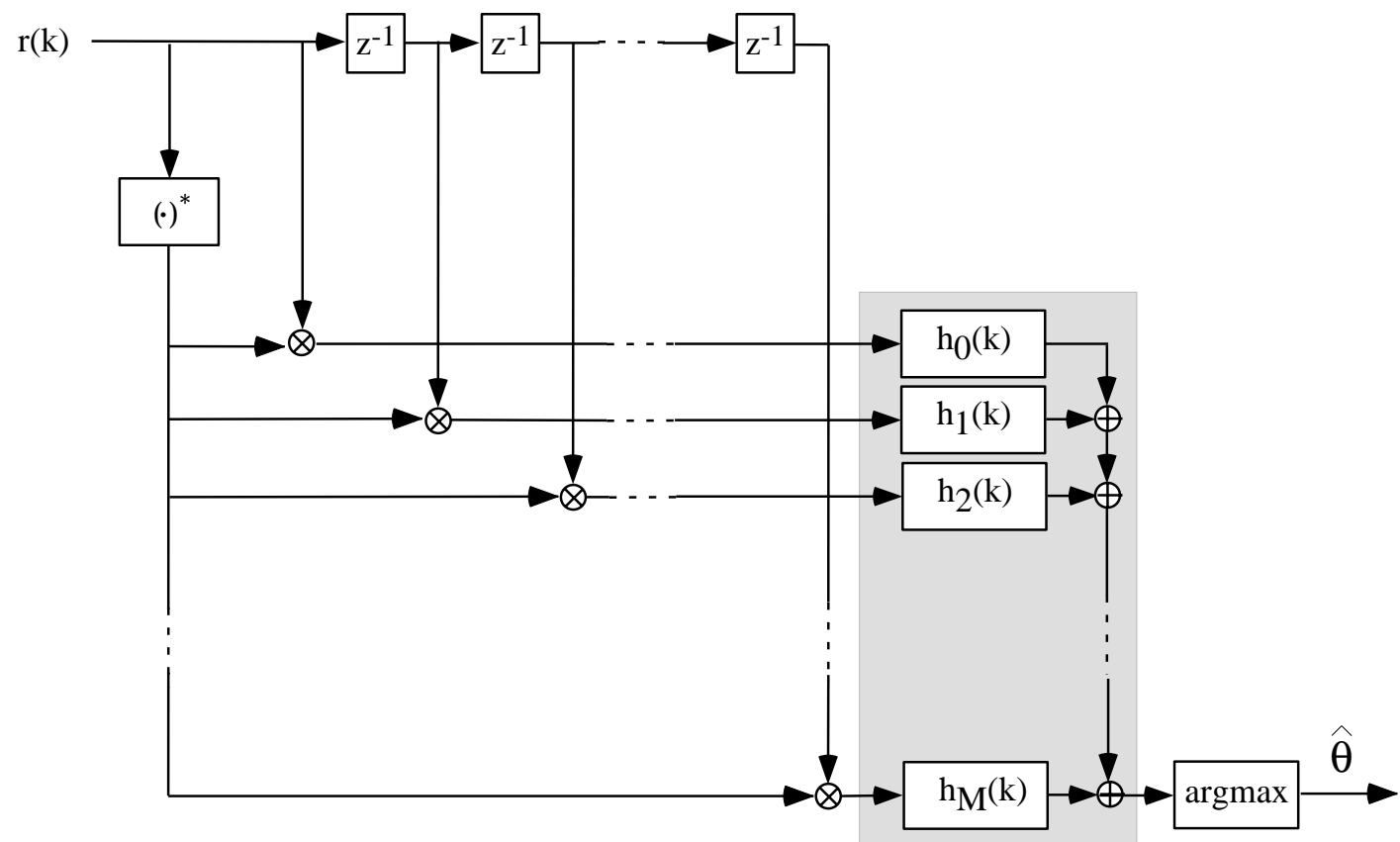

Figure 5: Structure of the estimator designed for a dispersive channel. 


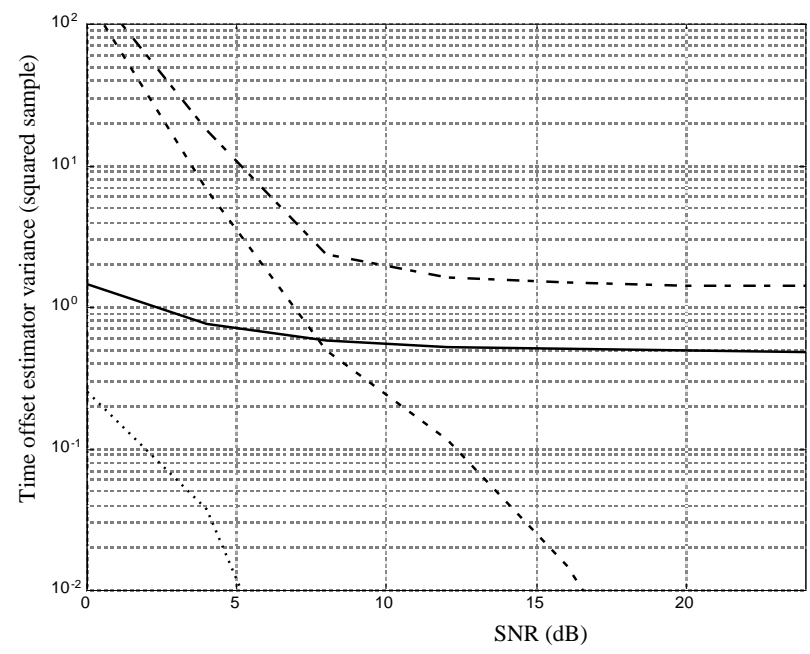

Figure 6: Variance of the time offset estimator in a system with pulse shaping. The estimator designed without pulse shaping with (solid) and without (dash-dotted) averaging over 10 symbols. The estimator designed for pulse shaping with (dotted) and without (dashed) averaging over 10 symbols.

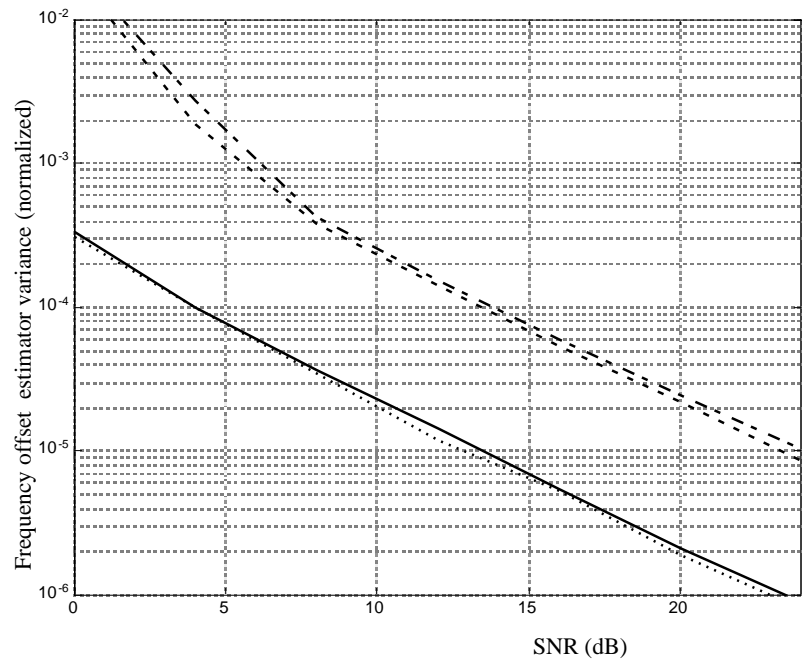

Figure 7: Variance of the frequency offset estimator in a system with pulse shaping. The estimator designed without pulse shaping with (solid) and without (dash-dotted) averaging over 10 symbols. The estimator designed for pulse shaping with (dotted) and without (dashed) averaging over 10 symbols. 


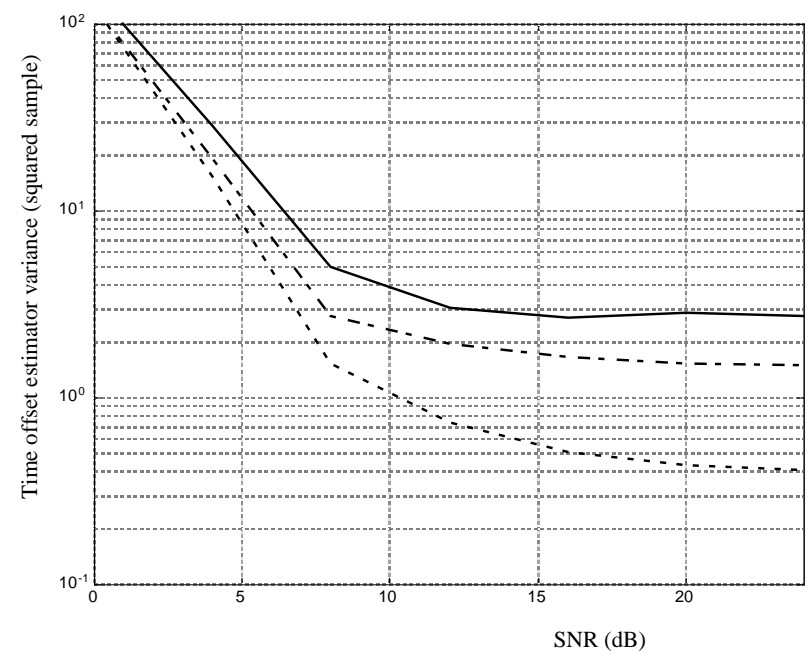

Figure 8: Variance of the time offset estimator for a system with pulse shaping and channel dispersion. The estimator designed for the AWGN channel (solid), the estimator designed with only the knowledge about the channel dispersion (dash-dotted), and the estimator designed with only the knowledge about the pulse shape (dashed).

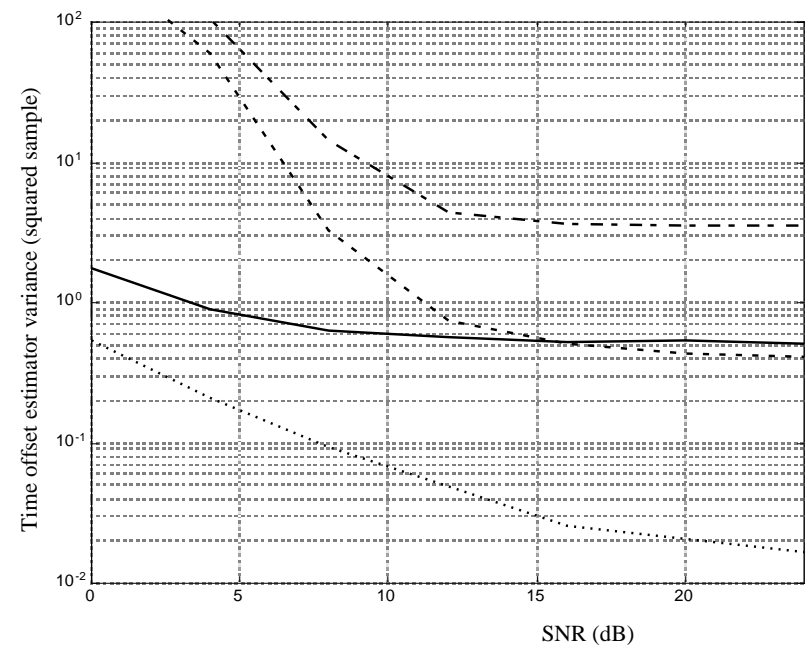

Figure 9: Variance of the time offset estimator in a system with pulse shaping and channel dispersion.The estimator designed for the AWGN channel with (solid) and without (dash-dotted) averaging over 10 symbols. The estimator designed for pulse shaping with (dotted) and without (dashed) averaging over 10 symbols. 


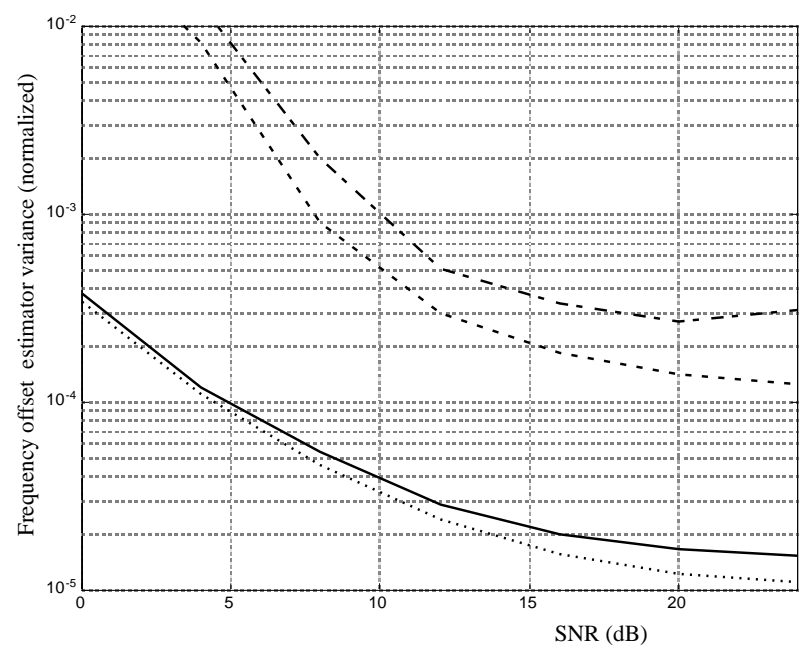

Figure 10: Variance of the frequency offset estimator for a system with pulse shaping and channel dispersion. The estimator designed for the AWGN channel with (solid) and without (dash-dotted) averaging over 10 symbols. The estimator designed for pulse shaping with (dotted) and without (dashed) averaging over 10 symbols. 